\title{
Flux and neutrino interaction model constraints using the T2K near detectors
}

\author{
Laura Zambelli*t \\ KEK, Japan \\ E-mail: lzambell@post.kek.jp
}

\begin{abstract}
Since 2014, the T2K long-baseline neutrino oscillation experiment in Japan has been running with reversed horn current to produce a beam enhanced in muon anti-neutrinos. Near detectors located 280 meters from the target allowed for a study of neutrino interactions prior to the onset of neutrino oscillations. By selecting muon (anti)-neutrino charged current interactions in various channels according to pion multiplicity, the neutrino flux and interaction model uncertainties are greatly reduced. In particular, the large contamination of neutrinos in the predominant antineutrino flux can be measured and constrained, a critical handle in the study of anti-neutrino oscillations at T2K. We present the results of a combined analysis of data from both neutrinoenhanced and antineutrino-enhanced running using an updated neutrino interaction model to incorporate multi-nucleon and other nuclear effects.
\end{abstract}

The European Physical Society Conference on High Energy Physics

22-29 July 2015

Vienna, Austria

*Speaker.

$\dagger$ on behalf of the $\mathrm{T} 2 \mathrm{~K}$ collaboration 


\section{The T2K experiment}

T2K (Tokai To Kamiokande) is a long baseline accelerator-based neutrino oscillation experiment located across Japan [1], as sketched in Fig 1. The main goal of the T2K experiment is to precisely measure the $\theta_{13}$ mixing angle through the observation of the $v_{\mu} \rightarrow v_{e}$ oscillations [2]. Subsequently, the accuracy of the $\theta_{23}$ mixing angle can be greatly improved by the measurement of the $v_{\mu}$ disappearance [3]. Also, various cross section measurements are conducted as well as exotic searches. In the light of the latest results on the mixing angles, T2K has recently started exploring the $\mathrm{CP}$ violation in the leptonic sector by comparing electron-neutrino and electron-antineutrino appearance results.

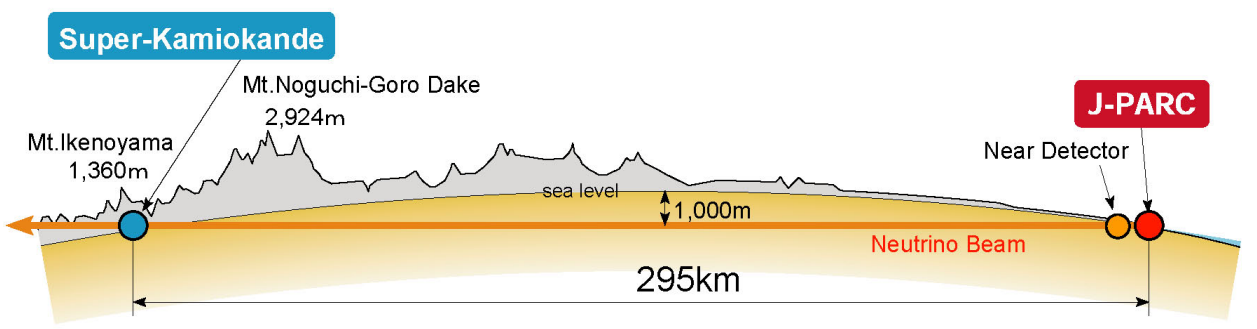

Figure 1: Schematic view of the T2K baseline

The J-PARC (Japan Proton Accelerator Complex) accelerator provides a $31 \mathrm{GeV}$ proton beam directed towards a long graphite target $\left(90 \mathrm{~cm}, 1.9 \times \lambda_{\text {int }}\right)$. A set of three horns - the target lies inside the first one - focuses (negatively) positively charged hadrons to produce the (anti-) neutrino enhanced flux, respectively. The following $\sim 96 \mathrm{~m}$ long decay tunnel allows the hadrons to decay ; a carbon-based beam dump at the end of the tunnel absords all other particles.

The near detector complex, located $280 \mathrm{~m}$ downstream the target, consists of two detectors. INGRID is placed exactly along the neutrino direction. It is made of 14 iron/scintillator modules arranged in a cross pattern. Its purpose is to control the direction and intensity of the neutrino flux. ND280 is located $2.5^{\circ}$ off-axis the neutrino beam direction. This multi-purpose detector is placed inside a $0.2 \mathrm{~T}$ magnet, which is recycled from the UA1 experiment. The main component of the ND280 is the tracker made of three TPCs and two fine grained detectors (FGDs). It measures the unoscillated neutrino flux, providing strong constraints on both the flux and neutrino cross section, reducing systematic uncertainties for the oscillation analysis.

The 50 kton water Cherenkov Super-Kamiokande (SK) is the far detector of the T2K experiment. It is located $295 \mathrm{~km}$ away from J-PARC, also off-axis at $2.5^{\circ}$. Muon and electron neutrinos can be reconstructed at SK thanks to the excellent electron/muon separation capability.

Placing detectors at a $2.5^{\circ}$ off-axis angle leads to a narrow band beam peaked at around $650 \mathrm{MeV}$. Compared to the on-axis flux, the beam is more intense at the peak energy, and the high energy tail is strongly suppressed. The off-axis angle, and peak energy, were chosen to maximizes the $v_{\mu} \rightarrow v_{e}$ oscillation probability at the far detector. At this energy, the main neutrino interaction channel is the charged-current quasi-elastic (CCQE) reaction : $v_{\mu}+n \rightarrow \mu^{-}+p$. 
The T2K experiment has started taking data in 2010. The beam power delivered by J-PARC has steadily increased since then, reaching now $371 \mathrm{~kW}$. For the analysis presented here, data from $6.57 \times 10^{20}$ protons on target (POT) in neutrino mode and $4.30 \times 10^{19}$ POT in anti-neutrino mode is used.

\section{Neutrino flux prediction tuned to hadroproduction data}

For clarity, the following section will explain the flux tuning in neutrino mode only. The tuning of the anti-neutrino mode flux is done in a similar same way.

When the proton interacts in the graphite target, several types of hadrons are produced. Along with the charged pions - main contributors of the $v_{\mu}$ flux - kaons, protons and neutral strange particles escape the target. The decay of those hadrons, together with the decay of muons produced together with the $v_{\mu}$, leads to the presence of an irreducible background flux of $v_{e}, \bar{v}_{\mu}$ and $\bar{v}_{e}$.
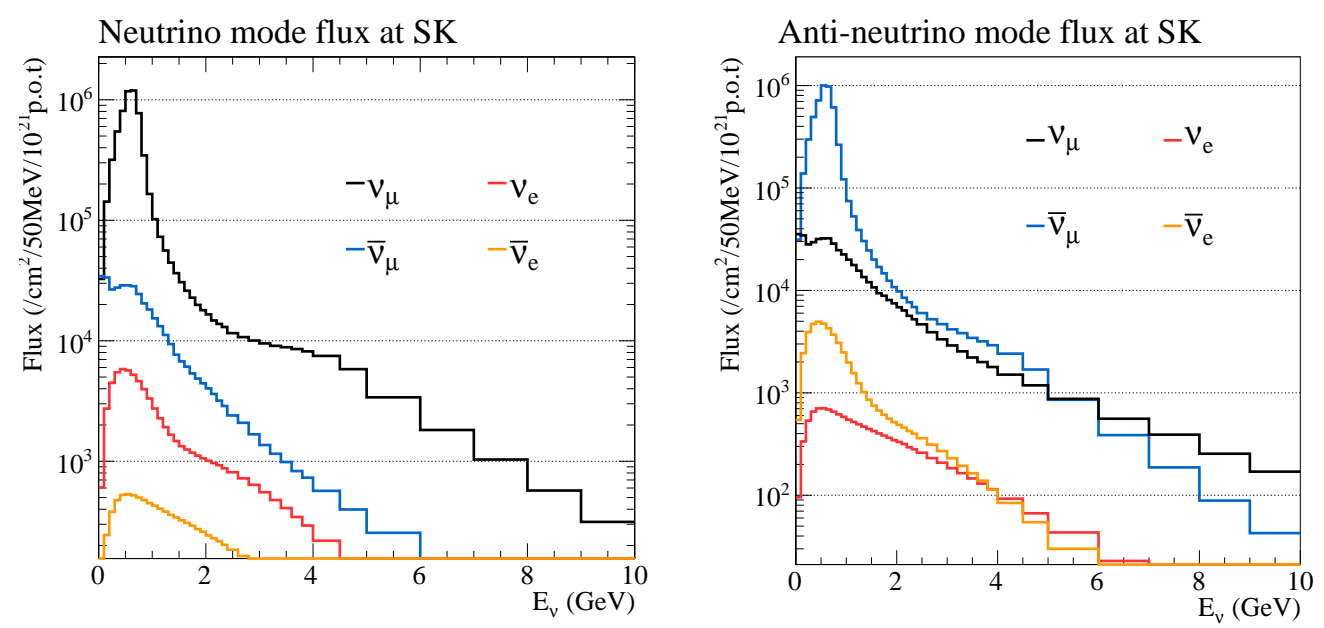

Figure 2: Tuned neutrino flux prediction at the far detector in neutrino mode (left) and anti-neutrino mode (right) normalized to $10^{21}$ POT.

The primary interaction of the proton on carbon leads to the production of about $60 \%$ of the $v_{\mu}$ and $v_{e}$ flux at the peak energy. Re-interactions in the target account for $30 \%$ of the flux. The remaining $10 \%$ are due to re-interactions in the beamline material [4]. To accurately predict the neutrino flux in the detectors, a good knowledge of the production cross section and kinematics of hadrons from proton-carbon interactions is mandatory. In order to tune the T2K neutrino flux prediction the hadroproduction data from an auxiliary experiment, NA61/SHINE [5], is used [6]. The fixed-target large acceptance spectrometer at CERN has collected data at the T2K proton beam energy with two different targets. A $2 \mathrm{~cm}\left(0.04 \times \lambda_{\text {int }}\right)$ thin target allows us to study the primary interactions of protons, while a $\mathrm{T} 2 \mathrm{~K}$ replica target provides informations on the re-interaction that occur in the long target. The thin target data taken in 2007 and 2009 enabled the measurement of the production cross section in proton-carbon interaction and the production spectra of charged pions, kaons, protons, $K_{S}^{0}$ and $\Lambda[7,8,9,10]$. Those data are currently used in the tuning process of the T2K flux. First, the flux is obtained in Monte Carlo simulations where interactions in the target are handled by Fluka [11]. The profile of the proton beam impining the target is reproduced 
according to the data. Propagation of all the particles escaping the target through the secondary beamline is simulated by GEANT3 using the GCALOR hadroproduction model. For every neutrino produced, most of the inelastic interactions in its history are tuned to the external data, mainly NA61/SHINE. The hadroproduction data can be scaled to lower momentum (using the Feynman scaling hypothesis) and/or to other targets. Moreover, we assume that Fluka reproduces accurately the production cross sections, hence only the GCALOR interaction cross sections are tuned to external data.

In Fig. 2 the tuned expected fluxes at the far detector are presented. It is important to notice the higher contamination of wrong sign neutrinos when the experiment runs in anti-neutrino mode. As the far detector cannot distinguish positively and negatively charged leptons, this background needs to be precisely understood.

The uncertainties on the flux are separated into two categories. Beamline-related uncertainties include those on the proton beam profile, the off-axis angle, the horn current, field and alignments between the proton beam, the target and the horns. Uncertainties associated with the tuning take into account the errors on the external data, the scaling of the data, the treatement of the re-interactions and finally the tuning of the secondary nucleon interactions. The total uncertainty on the flux at the peak energy has now decreased to the level of $9 \%$.

\section{Cross section models}

The neutrino interactions in the detectors are simulated with the NEUT Monte Carlo generator [12]. The recent upgrades of the generator includes addition of the multi-nucleon ejection channel (called MEC or 2p2h) on carbon and oxygen based on the Nieves model [13] with neutrino interacting with a pair of nucleons. This contribution can explain the higher event rate observed in the MiniBooNE CCQE measurement. The MEC events bias the energy reconstruction of CCQElike events. It is the first time that the MEC dynamics is taken into account in an oscillation analysis. In a conservative approach, no correlations between the carbon and oxygen MEC cross section are
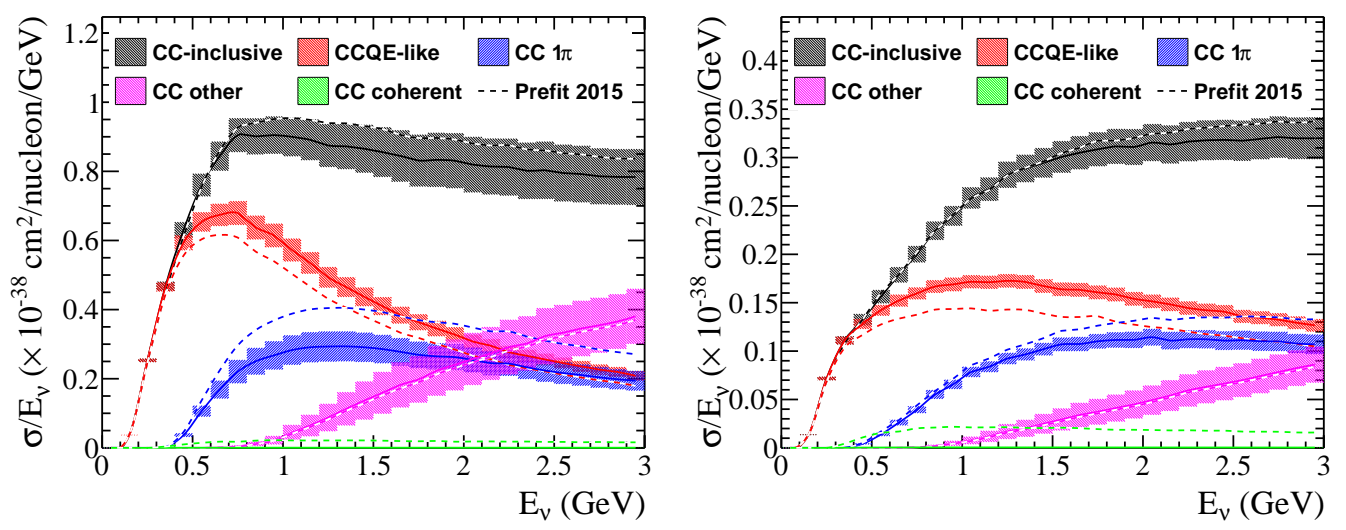

Figure 3: $v_{\mu}$ (left) and $\bar{v}_{\mu}$ (right) cross section predictions at the ND280 as a function of the neutrino energy (divided by the neutrino energy). Dashed lines represent the central previous predictions for comparisons. CCQE-like means no pion in the final state.

assumed. 
The T2K collaboration decided to use the relativistic Fermi gas model together with the random phase approximation corrections as its CCQE model instead of the NEUT default one. The nominal NEUT cross section parameters, such as the axial mass, the Fermi momentum or the MEC normalisation are then adjusted to external CCQE data. As tensions with some data appeared, errors on the CCQE cross section models uncertainties are inflated.

The resonant pion production has been retuned in NEUT. Graczyk and Sobczyk [14] form factors are now used, and the ANL and BNL bubble chamber dataset has been re-analyzed [15]. Other interaction channels like deep-inelastic scattering or neutral current interactions are mostly unchanged compared to our previous results. In Fig. $3 v_{\mu}$ and $\bar{v}_{\mu}$ cross section predictions as functions of neutrino energy are presented.

\section{Flux and cross section constraints}

The flux prediction and the cross section parameters described previously are finally constrained using the data from the ND280 tracker. Muon (anti)-neutrino events are selected by requesting an interaction vertex to be in the first FGD, and that the attached track is crossing the TPC reconstructed consistently with a (positive) negative muon hypothesis. The events are then divided into several samples. For $v_{\mu}$ interactions in the neutrino mode, 3 samples are made depending on the number of tagged pions : no pions (CCQE-like sample), 1 pion or more than 1 pion. As the anti-neutrino mode data suffers from a lack of statistics, only two samples are made : with one or more tracks crossing the TPCs. This categorization is done for both $v_{\mu}$ and $\bar{v}_{\mu}$ interactions.
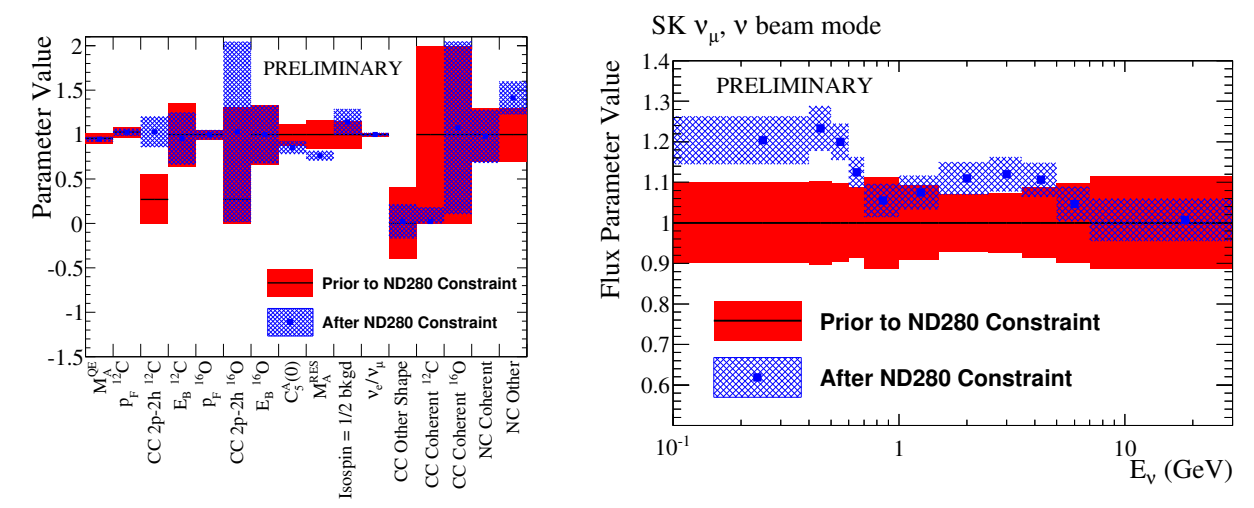

Figure 4: Before (red) and after (blue) the ND280 constraint for the cross section (left) and far detector flux parameters (right) with their corresponding uncertainties.

A binned likelihood (in bins of muon momentum and angle) is then adjusted to these samples. The fitting process takes into account the variation of the flux, the cross section model and the near detector uncertainties together with their correlations. The data constrained flux and cross section parameters are then extracted from the fit. In Fig. 4 variations on the cross section and flux parameters central values and uncertainties are presented. In general, the uncertainties on the parameters decrease significantly as compared to their prior values.

For the $\bar{v}_{\mu}$ disappearance analysis, the uncertainties related to the flux and the cross section are reduced from $9.2 \%$ to $3.4 \%$. As stated previously, the MEC cross section on oxygen remains as 
not correlated to carbon. The total uncertainty for the oscillation analysis is now $11.6 \%$, to be compared with $14.4 \%$ without the ND280 constraints.

\section{Conclusions}

Using the NA61/SHINE hadroproduction data, recent developments in the modeling of neutrino interactions and the ND280 data, the T2K collaboration managed to reduce uncertainties for the $\bar{v}_{\mu}$ disappearance analysis [16] to the level of $11.6 \%$. Further improvements are expected in the near future. The tuning of the flux with the help of the 2009 NA61/SHINE replica target data [17] will allow for a a better treatment of the re-interactions happening in the target. As up to $90 \%$ of the flux can be directly constrained to external data, a significant reduction of the uncertainties is expected.

A better understanding of the MEC effect should improve the cross sections predictions. Once new ND280 samples will be added to the analysis, in particular neutrino interactions in the second FGD filled with water bags, will allow to further decrease the uncertainties.

\section{References}

[1] K. Abe et al. [T2K Collaboration] Nucl. Instrum. Meth. A 659 (2011) 106-135

[2] K. Abe et al. [T2K Collaboration], Phys. Rev. Lett. 112 (2014) 061802

[3] K. Abe et al. [T2K Collaboration], Phys. Rev. D 91 (2015) 7, 072010

[4] N. Abgrall et al. [NA61/SHINE Collaboration], Nucl. Instrum. Meth. A 701 (2013) 99

[5] N. Abgrall et al. [NA61/SHINE Collaboration], JINST 9 (2014) P06005

[6] K. Abe et al. [T2K Collaboration], Phys. Rev. D 87 (2013) 1, 012001

[7] N. Abgrall et al. [NA61/SHINE Collaboration], Phys. Rev. C 84 (2011) 034604

[8] N. Abgrall et al. [NA61/SHINE Collaboration], Phys. Rev. C 85 (2012) 035210

[9] N. Abgrall et al. [NA61/SHINE Collaboration], Phys. Rev. C 89 (2014) 2, 025205

[10] N. Abgrall et al. [NA61/SHINE Collaboration], CERN-PH-EP-2015-278, arXiv: 1510.02703, submitted to EPJ C

[11] T.T. Böhlen et al. Nuclear Data Sheets 120, 211-214 (2014)

[12] Y. Hayato, Nucl. Phys. (Proc. Supp.) 112, 171 (2002)

[13] J. Nieves, I. Ruiz Simo and M. J. Vicente Vacas, Phys. Rev. C 83 (2011) 045501

[14] K. M. Graczyk and J. T. Sobczyk, Phys. Rev. D 77 (2008) 053001

[15] C. Wilkinson, P. Rodrigues, S. Cartwright, L. Thompson and K. McFarland, Phys. Rev. D 90 (2014) 11,112017

[16] M. Ravonel, EPS-HEP 2015 proceeding

[17] A. Haesler, PhD thesis, University of Geneva, CERN-THESIS-2015-103. 